\title{
Review Article \\ Inflammation, Iron, Energy Failure, and Oxidative Stress in the Pathogenesis of Multiple Sclerosis
}

\author{
Lukas Haider \\ Department of Neuroimmunology, Center for Brain Research and Department of Biomedical Imaging and Image-Guided Therapy, \\ Medical University of Vienna, Währinger Gürtel 18-20, 1090 Vienna, Austria
}

Correspondence should be addressed to Lukas Haider; lukas.haider@meduniwien.ac.at

Received 8 March 2015; Revised 6 May 2015; Accepted 18 May 2015

Academic Editor: Vincent Pialoux

Copyright (C) 2015 Lukas Haider. This is an open access article distributed under the Creative Commons Attribution License, which permits unrestricted use, distribution, and reproduction in any medium, provided the original work is properly cited.

\begin{abstract}
Multiple sclerosis is a chronic inflammatory demyelinating disease of the central nervous system. Different trigger pathologies have been suggested by the primary cytodegenerative "inside-out" and primary inflammation-driven "outside-in" hypotheses. Recent data indicate that mitochondrial injury and subsequent energy failure are key factors in the induction of demyelination and neurodegeneration. The brain weighs only a few percent of the body mass but accounts for approximately $20 \%$ of the total basal oxygen consumption of mitochondria. Oxidative stress induces mitochondrial injury in patients with multiple sclerosis and energy failure in the central nervous system of susceptible individuals. The interconnected mechanisms responsible for free radical production in patients with multiple sclerosis are as follows: (i) inflammation-induced production of free radicals by activated immune cells, (ii) liberation of iron from the myelin sheets during demyelination, and (iii) mitochondrial injury and thus energy failure-related free radical production. In the present review, the different sources of oxidative stress and their relationships to patients with multiple sclerosis considering tissue injury mechanisms and clinical aspects have been discussed.
\end{abstract}

\section{Introduction}

Multiple sclerosis is a chronic disease of the central nervous system and is associated with the formation of focal myelin loss and progressive neurodegeneration [1].

Clinically, $80 \%$ of patients with multiple sclerosis present with relapsing-remitting multiple sclerosis (RRMS), which refers to clearly defined episodes of neurological dysfunction followed by (partial) recovery. After 15-25 years, RRMS is transformed into secondary progressive multiple sclerosis (SPMS) in a majority of the patients; SPMS is characterised by progressive neurological symptoms. Patients with multiple sclerosis (10-15\%) enter this neurodegenerative phase at disease onset, which is referred to as primary progressive multiple sclerosis (PPMS) [2-4].

Once patients enter the progressive phase, no currently available drug provides a significant clinical effect. Neurological decline in these patients is caused by chronic and diffuse neurodegeneration. Neurodegeneration is closely associated with inflammation, both morphologically and quantitatively, in all phases of multiple sclerosis $[5,6]$. Anti-inflammatory drugs that fail to prevent disease progression are highly effective in reducing neuroinflammatory attacks in patients with RRMS. No animal models are available that mimic this enigma of the progressive phase and three nonexclusive hypotheses have been proposed as follows [7]:

(i) Inflammation in the relapsing-remitting and progressive phases is driven by the same mechanisms, but during progressive MS the central nervous system does not respond to currently available antiinflammatory drugs [5], which may be caused by the closed blood-brain barrier present in progressive MS $[8,9]$.

(ii) Microglia, which are under the control of intact neurons, may become chronically active due to primary neurodegeneration, axonal degeneration, and additional peripheral activation processes such as systemic inflammation [10-13].

(iii) Multiple sclerosis may be caused by primarily cytodegenerative processes/infections, which are amplified by inflammation [14-17]. 
Although these models are fundamentally different, they share a common feature; that is, the tissue injury mechanisms are closely related to the production of reactive oxygen and nitrogen species.

\section{Sources of Oxidative Stress in Multiple Sclerosis}

The central role of oxidative stress has been frequently suggested in the pathogenesis of multiple sclerosis [18] based on the biochemical analysis of cerebrospinal fluid/blood samples, tissue homogenates [19-22], and animal models of multiple sclerosis $[23,24]$. Oxidised DNA molecules, lipids, and protein adducts are frequently found in active multiple sclerosis lesions $[24,25]$ and are associated spatially and quantitatively with apoptotic oligodendrocytes and neurodegeneration in the brains of patients with multiple sclerosis [26].

\section{Inflammation}

Active inflammation and breakdown of the blood-brain barrier can be detected in the brains of patients with multiple sclerosis as gadolinium enhancing magnetic resonance imaging lesions [9, 27-29]. Although inflammatory infiltrates are present in all the stages of multiple sclerosis [5], the qualitative and quantitative composition is variable and dependent on clinical parameters (age, disease duration) and their location within the brain (meningeal, perivascular, and parenchymal). The diverse aspects of inflammation in patients with multiple sclerosis are discussed extensively [30]. Two waves of inflammatory responses can be distinguished as follows: (i) The initial/prephagocytic lesions contain few CD8 positive T-cells [17] and tissue injury is associated with the presence of activated microglia that infiltrate the parenchyma [31]; (ii) this is followed by the secondary recruitment of T-cells, Bcells, and macrophages during myelin breakdown [30].

Macrophage/microglia-derived reactive oxygen and nitrogen species trigger axonal injury [23]. The enzymes involved in the production of free radicals as well as their regulatory and catalytic subunits are upregulated in active multiple sclerosis lesion areas [32-35], where oxidative stress adducts are found most frequently $[26,36]$. Experimental autoimmune encephalomyelitis (EAE) is an animal model that mimics certain phenomena observed in patients with multiple sclerosis [37]. The gene of Gp91phox encodes the catalytic component of NADPH oxidase [38]. Mice deficient in Gp91phox develop a milder form of EAE [39].

In physiological conditions, neurons, astrocytes, and oligodendrocytes express molecules that bind to receptors that are expressed on microglia. Signalling via these molecules, such as the fractalkine receptor ligand CX3CL1 [10], the membrane glycoprotein CD200 [40], the integrin associated protein myelin CD47 [41], and sialic acid alpha2,8-linked polysialic acids [42], inhibits microglial activation [43]. Axonal degeneration can thus activate microglia that are distant from the initial site of neuronal injury due to the decreased expression of these molecules [13], which may trigger the microglia-mediated removal of debris and delivery of neurotrophic factors. Similar to other neurodegenerative diseases, such "preactivated" microglia can be more easily converted into a cytotoxic form when exposed to a proinflammatory cytokine milieu (e.g., via systemic inflammations) [44]. In addition to the randomly distributed plaques, the preactivation of microglia in EAE using Wallerian degeneration determined the location of demyelinating lesions that appeared in the ipsilateral thalamus after cortical cryoinjury and in the ipsilateral optic nerve, contralateral optic tract, and superior colliculus after unilateral eye ball enucleation [45]. Accordingly, cortical thickness in patients with multiple sclerosis is associated with the connected thalamic nucleus's neuronal cell density and the myelin content of the anatomical connection [12].

In patients with long disease durations (median $>372$ months), the level of inflammatory infiltrates is similar to those found in age-matched controls [5]. Although there is a close association between oxidative stress and inflammation in active lesions and patients with RRMS, it is also pronounced in progressive MS $[26,46]$, which raises the question whether additional reactive oxygen and nitrogen species are present in patients with progressive MS.

\section{Iron}

In the human brain, iron is primarily stored by ferritin [47] in the myelin sheets $[48,49]$. It accumulates physiologically with age, reaching a plateau at 40-50 years, depending on the anatomical structure that is analysed [50]. Experimental spinal cord injury revealed that iron can amplify oxidative damage in lesions of the central nervous system [51]. Myelin breakdown and subsequent phagocytosis of myelin debris occur at active multiple sclerosis lesions $[17,30]$. It has been suggested that iron liberated into the extracellular space during the course of myelin breakdown amplifies the first wave of oxidative stress in multiple sclerosis lesions [52]. Finally, iron is taken up by macrophages and microglia. However, these cells degenerate $[52,53]$ and thus release their iron content into the extracellular space, which initiates an additional wave of oxidative stress [54,55].

Clinically, the duration of the relapsing-remitting phase can vary between individuals [3]; however, the coerciveness of the progressive phase and the rate of neurological decline are highly consistent irrespective of the preceding disease course or its severity. This led to the assumption that neurodegenerative mechanisms in patients with progressive MS depend on the patients' age $[4,56]$. Iron accumulates in the human brain in an age-dependent manner and ironmediated amplification of oxidative stress may contribute to the age-related pathology of progressive MS [1,57]. Iron accumulation is scarce in rodent models of multiple sclerosis and barely reflects the extent of oxidative injury observed in patients with multiple sclerosis [37].

In the human brain, the highest iron content is measured in the deep grey matter nuclei, particularly in the basal ganglia $[50,52,58]$. However, lesion incidence and destructiveness are similar in the deep grey matter nuclei and the white matter of patients with multiple sclerosis $[46,59]$. 


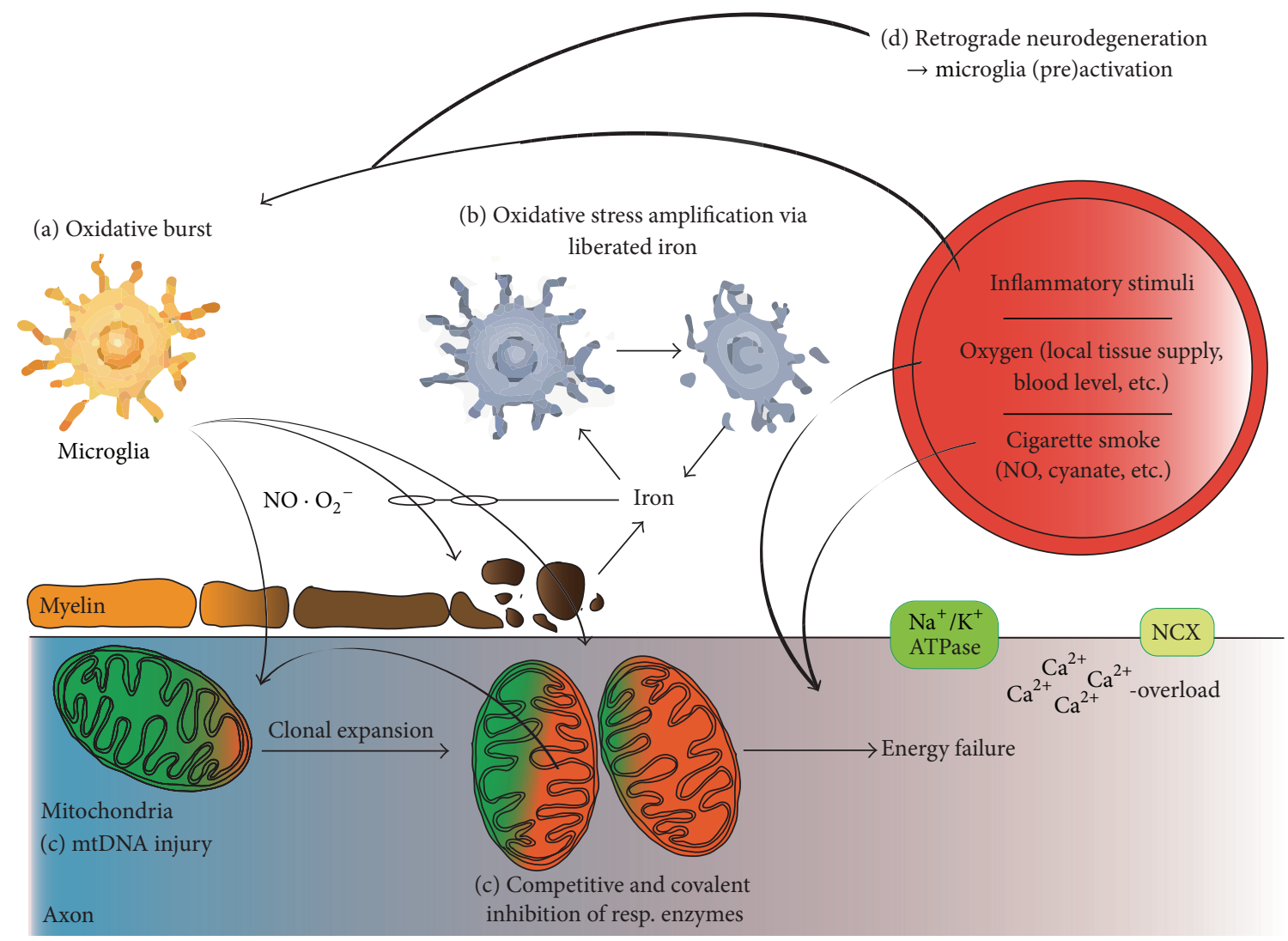

FIGURE 1: Oxidative stress-related mechanisms of tissue injury in multiple sclerosis. The temporal sequence and interconnection of cytotoxic events in MS may be different in individual lesions, stages of the disease, and different patients. Therefore, Figure 1 does not describe a timeline from events depicted in (a) to that in (d). (a) Microglia are activated by an unknown trigger pathology, the breakdown of the blood-brain barrier, and local and systemic inflammatory stimuli. Microglia activation itself may further impair the blood-brain barrier permeability. Microglia release nitric monoxide and superoxide molecules into the extracellular space. Nitric monoxide is uncharged and therefore penetrates lipid layers. Contrary to nitric monoxide, superoxide is unable to diffuse across biological membranes. It is rapidly converted into hydrogen peroxide, which, in contrast to superoxide, is able to diffuse into the extracellular environment. Additional amplification mechanisms involve microglia preactivation via axonal degeneration (d). (b) Iron is physiologically stored within the myelin sheets and liberated into the extracellular space upon demyelination. Extracellular iron amplifies oxidative stress as it travels between the ferrous and ferric states, inducing the production of highly reactive hydroxyl radicals. Iron is absorbed by microglia, which show histological signs of cell death under the high iron load and thus may release iron and initiate a second wave of oxidative stress. (c) Mitochondrial DNA (mtDNA) is vulnerable to free radical-mediated damage resulting in mtDNA deletions, which are found in neurons and axons of patients with multiple sclerosis. Mitochondria carrying such mutations are amplified by the clonal expansion in neurons. The mitochondrial respiratory chain is inhibited by covalent modifications caused by free radicals both competitively and irreversibly. A combination of these factors leads to energy failure via decreased ATP production. An important source of free radicals is the mitochondrial respiratory chain itself, particularly at low oxygen tension and reperfusion and in demyelinated axons. The exogenous factors, such as free radicals and cyanate delivered by smoking, inhibit mitochondrial function and cause demyelination in experimental conditions. Energy deficiency lowers $\mathrm{Na}^{+} / \mathrm{K}^{+}$-ATPase activity, resulting in the reverse operation of the $\mathrm{Na}^{+} / \mathrm{Ca}^{2+}$ exchanger (NCX) and thus increases $\mathrm{Ca}^{2+}$ levels. This event further activates the neurodegenerative and cell death pathways.

Histologically, the deep grey matter of patients with multiple sclerosis reveals a diffuse neurodegeneration pattern [60], quantitatively related to the patients' motor dysfunction prior to death [46]. The deep grey matter lesions of patients with multiple sclerosis show high levels of oxidative stress in neurons and oligodendrocytes. The level of oxidative stress in these lesions is positively associated with local iron load from both a topographical and a quantitative point of view $[46,52]$. Similar to white matter lesions, two waves of iron liberation (Figure 1) have been suggested [46]. Inducible nitric oxide synthase (iNOS) is not expressed in the human central nervous system in baseline conditions [61, 62]. It is remarkable that the level of iNOS is elevated in the deep grey matter nuclei compared to the cortex or white matter in both patients with multiple sclerosis and controls [46]. Nitric monoxide is a competitive inhibitor of the respiratory chain $[63,64]$. In high concentrations, in hypoxic conditions, or in the presence of superoxide, nitric monoxide promotes excitotoxicity and apoptosis $[65,66]$. Low iNOS expression is cytoprotective in normoxic conditions and in the absence of superoxide [67]. In iron laden microglia cells, iNOS expression is upregulated $[68,69]$. These findings suggest that iNOS upregulation could 
be an adapting mechanism in response to iron accumulation in the central nervous system and particularly in the deep grey matter [70]. Nitric monoxide mediated inhibition of the respiratory enzymes may also explain the clinical and pathological observation of early atrophy of deep grey matter in patients with Alzheimer's disease $[71,72]$ and that the deep grey matter nuclei are selectively vulnerable to hypoxemia or energy failure, in conditions such as carbon monoxide poisoning [73] and mitochondriopathies [74].

\section{Hypoxia and Energy Failure}

5.1. Mitochondrial Injury. The role of the mitochondria in the pathogenesis of multiple sclerosis was suggested by the observation that patients with Leber's hereditary optic neuropathy, a disease caused by mitochondrial DNA mutations, have an increased risk of developing multiple sclerosis-like Harding's disease [75]. In patients with multiple sclerosis, mitochondrial dysfunction has been described extensively in the cortex and white matter $[1,76]$. Hypoxia-like lesions (pattern III multiple sclerosis lesions) [77] display various mitochondrial respiratory chain defects in axons, oligodendrocytes, and astrocytes [78]. Mitochondrial gene expression of 26 nuclearencoded subunits of the oxidative phosphorylation chain and activity of complexes I and III are decreased in cortical neurons. The distribution of these "respiratory deficient" neurons is unknown, but they are not restricted to the areas of myelin loss [79, 80], suggesting a diffuse process. Reactive oxygen and nitrogen species are produced by activated microglia and macrophages through oxidative burst that releases these molecules into the extracellular space [81]. Nitric monoxide can diffuse across membranes and competes with oxygen for the binding site of mitochondrial cytochrome c oxidase; thus, it decreases respiratory chain function [82]. Reactive oxygen and nitrogen species induce covalent modifications and thus mutations in the mitochondrial DNA, which is more vulnerable than nuclear DNA [74, 83]. Mitochondrial DNA defects are present in patients with multiple sclerosis $[80,84]$. Mitochondrial DNA mutations induced by reactive species inhibit the efficiency of oxidative phosphorylation and further increase the production of reactive oxygen species, thus leading to a vicious circle [85]. In normal conditions, $1-2 \%$ of the electrons escape from mitochondrial oxidative phosphorylation [86] and react with molecular oxygen producing superoxides in the mitochondrial matrix, in the compartment that contains mitochondrial DNA [87]. Hypoxia increases the production of reactive oxygen intermediates by deregulating the mitochondrial electron transport chain [88, 89]. Similarly, mitochondrial superoxide production increases when adenosine triphosphate (ATP) production is decreased (e.g., at a high membrane potential and $\mathrm{pH}$ gradient, low levels of coenzyme Q, and high nicotinamide adenine dinucleotide (NAD)/NAD+ ratio) $[90,91]$. These conditions are present in demyelinated axons with an impaired conduction of saltatory axon potential transmission [18].

The detoxification of reactive oxygen species reversed mitochondrial and axonal injury in experimental settings [23], where the nitration of mitochondrial proteins precedes tissue injury and is present in the intact axons of animals with EAE [92]. In patients with multiple sclerosis, the proportion of mutant to wild-type mitochondrial DNA copies (heteroplasmy) in metabolically active postmitotic neurons increases with disease progression and increases age, via the clonal expansion of defective mitochondrial DNA [93]. Damaged mitochondria are normally removed from the cells by autophagy, which involves the formation of doublemembrane structures called autophagosomes that fuse with lysosomes to degrade their content [94]. The autophagosome formation is inhibited by mTOR-dependent signalling pathways [94]. mTOR signalling is inhibited when nutrients are scarce, growth factor-related signalling is reduced, and ATP concentrations are low. In these situations, mTOR signalling is suppressed and biogenesis of the autophagosomes increases [95]. The reason why mitochondria with mutated mitochondrial DNA undergo clonal expansion and whether this process is related to changes in mTOR signalling in multiple sclerosis remains unknown [1].

Mitochondrial dysfunction and consequently ATP deficiency in multiple sclerosis lesions lead to the failure of sodium removal from the axoplasm into the extracellular space during action potential conduction [56]. In this condition, accumulated sodium is replaced by calcium ions by the reverse operation of the sodium-calcium exchanger (NCX). Calcium subsequently activates calpains, which initiate the proteolytic degeneration of cytoskeletal proteins $[16,96$, 97]. In addition, aberrantly expressed voltage-gated calcium channels and glutamate receptors have been described in multiple sclerosis lesions, and their presence might amplify calcium toxicity $[98,99]$. The ionic imbalance may be amplified by alterations in individual sodium channel subunits in multiple sclerosis lesions $[100,101]$.

5.2. Real/Virtual Hypoxia. The central nervous system is highly dependent on the continuous blood flow and mitochondrial metabolism that produces ATP [102]. This dependence is well illustrated by the large number of neurological disorders due to genetic alterations in mitochondrial and nuclear genes encoding mitochondrial proteins [74] and CNS injury in conditions of hypoxia or hypoglycaemia [73]. The hypoxic features of multiple sclerosis lesions including the expression of HIF-1-alpha, other hypoxia-related proteins, and elevated concentrations of lactate within lesions have been reported [103-110]. In patients with multiple sclerosis, diverse factors related to local oxygen supply/demand contribute to the endpoint of neurodegeneration. Brain inflammation reduces the oxygen supply due to oedematous tissue swelling [111, 112] and increases oxygen consumption by the presence of inflammatory infiltrates [113] and by the formation of a diffusion barrier to oxygen. The neurological deficits in animals with EAE are correlated with spinal cord white and grey matter hypoxia quantitatively, temporally, and spatially [113]. Similar findings are suggested in humans with multiple sclerosis. Brownell and Hughes determined the location of 1594 macroscopically visible plaques and reported "the peculiarity that these are situated on the boundary zones between major cerebral arteries, which have penetrated in 
this periventricular region to their further point of supply" [114]. These border zones between the major cerebral arteries (the so-called watersheds) have a decreased oxygen tension, and magnetic resonance imaging of 1249 patients with multiple sclerosis revealed high levels of lesion load in these watershed zones [115], which is consistent with previous findings [116]. A meta-analysis of voxel-based morphometric studies in patients with multiple sclerosis revealed that the cortex located within watershed areas is more severely affected by atrophy than other cortical regions [117]. However, the relative contribution of axonal degeneration and subcortical lesion frequency on primary neurodegeneration compared to other factors such as meningeal inflammation and cortical lesion formation in these cortical areas remains unknown.

Smoking can trigger multiple sclerosis, propagate disease progression, and transiently worsen the motor functions in patients with multiple sclerosis [118-120]. Cigarette smoke contains over 4500 potentially toxic components including reactive oxygen species, nitric monoxide, and cyanate $[121,122]$. Cyanate inhibits the mitochondrial respiratory chain [123] and causes demyelination [124-126]. The exogenous inhibition of mitochondrial function via hypoxia and increased blood levels of cyanate and free radicals due to cigarette smoke inhalation may explain this environmental risk factor in the pathogenesis of multiple sclerosis; however, further experiments are warranted for confirmation.

\section{Clinical Consequences}

Energy failure, due to mitochondrial injury and oxidative stress, is a key player in the pathogenesis of multiple sclerosis. Early clinical research argued over the "relief by flush" therapy $[127,128]$. Drugs such as histamine and amyl nitrite, which increase perfusion and thus may counteract energy failure, provided only temporary beneficial effects [129]. Similarly, hyperbaric oxygen has highly significant transient effects in the treatment of patients with multiple sclerosis but failed to show any lasting results (e.g., 100\% oxygen at 2 atmospheres for 90 min once daily, for a total of 20 exposures) [130,131]. The failure of hyperbaric oxygen therapy in patients with multiple sclerosis may be related to oxidative stress, which can increase in such conditions, similar to reperfusion injury [132] and the temporal timing of oxygen delivery in oxygen-sensitive periods [113]. A novel approach targets histotoxic "virtual" hypoxia by counteracting mitochondrial injury [76]. These drugs may be beneficial for patients with progressive MS because they may be able to cross the relatively intact blood-brain barrier [133]. The potential targets for boosting mitochondrial functions are substances that would particularly enhance the operation of peroxisome proliferator-activated receptor gamma coactivator 1 alpha (PGC1-alpha) [134, 135]. PGC1-alpha is a transcriptional cofactor, which binds and activates nuclear transcription factors that are involved in mitochondrial function. In addition, PGC1-alpha expression is reduced in the cortical neurons of patients with multiple sclerosis [136]. Cyclophilin D and p66ShcA are both involved in the formation of mitochondrial permeability transition pores and subsequent cell death signalling. Cyclophilin D and p66ShcA inactivation significantly reduced axonal damage in EAE $[137,138]$. The gene delivery of superoxide dismutase 2 , a mitochondrial scavenger of superoxides, ameliorates the axonal pathology in EAE [139]. Similar protective properties were reported for MitoQ, an antioxidant accumulating in the mitochondria $[113,140]$. The anti-inflammatory drugs may penetrate the relatively intact blood-brain barrier and inhibit proinflammatory mediators that are released by $\mathrm{T}$ and B-lymphocytes or microglia $[1,5,8]$.

A conclusion, which genetic, experimental, and pathological investigations clearly suggest, is that there is no evidence for a single cause and thus therapeutic target of multiple sclerosis. Instead, multiple amplification steps orchestrate the clinically observed phenotype in susceptible individuals [141], which is well reflected in clinical trials. Drugs that target general or various inflammatory pathways such as blood-brain barrier permeability and immune suppression/modulation or combine additional cytoprotective properties have proven highly effective in the treatment of patients with multiple sclerosis [142-147]. It is important to note that the pharmacodynamics of such drugs may be highly specific (e.g., natalizumab blocks the VLA alpha-4 subunit); however, they interfere with the biological pathways that have very broad/unspecific effects on the organism (e.g., natalizumab prevents leucocytes from entering the central nervous system). The treatment strategies interfering with more specific downstream pathways proved not only less effective but also potentially dangerous [148-151]. The testing of new treatment regimes, particularly in clinical trials, may therefore benefit from combined approaches targeting different cell death pathways. Such approaches may involve anti-inflammatory therapy [1], protection against oxidative stress [152], mitochondrial injury [76], and hypoxic energy failure [113].

\section{Conflict of Interests}

There is no conflict of interests to declare in relation to this paper.

\section{Acknowledgment}

The author would like to thank Professor Hans Lassmann for the critical review of the paper.

\section{References}

[1] D. H. Mahad, B. D. Trapp, and H. Lassmann, "Pathological mechanisms in progressive multiple sclerosis," The Lancet Neurology, vol. 14, no. 2, pp. 183-193, 2015.

[2] A. Scalfari, A. Neuhaus, M. Daumer, P. A. Muraro, and G. C. Ebers, "Onset of secondary progressive phase and longterm evolution of multiple sclerosis," Journal of Neurology, Neurosurgery and Psychiatry, vol. 85, no. 1, pp. 67-75, 2014.

[3] A. Compston and A. Coles, "Multiple sclerosis," The Lancet, vol. 372, no. 9648, pp. 1502-1517, 2008.

[4] C. Confavreux, S. Vukusic, T. Moreau, and P. Adeleine, "Relapses and progression of disability in multiple sclerosis," 
The New England Journal of Medicine, vol. 343, no. 20, pp. 1430$1438,2000$.

[5] J. M. Frischer, S. Bramow, A. Dal-Bianco et al., "The relation between inflammation and neurodegeneration in multiple sclerosis brains," Brain, vol. 132, no. 5, pp. 1175-1189, 2009.

[6] R. Magliozzi, O. W. Howell, C. Reeves et al., "A Gradient of neuronal loss and meningeal inflammation in multiple sclerosis," Annals of Neurology, vol. 68, no. 4, pp. 477-493, 2010.

[7] B. D. Trapp and K.-A. Nave, "Multiple sclerosis: an immune or neurodegenerative disorder?" Annual Review of Neuroscience, vol. 31, pp. 247-269, 2008.

[8] S. Hochmeister, R. Grundtner, J. Bauer et al., "Dysferlin is a new marker for leaky brain blood vessels in multiple sclerosis," Journal of Neuropathology \& Experimental Neurology, vol. 65, no. 9, pp. 855-865, 2006.

[9] O. Ciccarelli, F. Barkhof, B. Bodini et al., "Pathogenesis of multiple sclerosis: insights from molecular and metabolic imaging," The Lancet Neurology, vol. 13, no. 8, pp. 807-822, 2014.

[10] A. E. Cardona, E. P. Pioro, M. E. Sasse et al., "Control of microglial neurotoxicity by the fractalkine receptor," Nature Neuroscience, vol. 9, no. 7, pp. 917-924, 2006.

[11] S. G. Meuth, O. J. Simon, A. Grimm et al., "CNS inflammation and neuronal degeneration is aggravated by impaired CD200CD200R-mediated macrophage silencing," Journal of Neuroimmunology, vol. 194, no. 1-2, pp. 62-69, 2008.

[12] J. Kolasinski, C. J. Stagg, S. A. Chance et al., "A combined post-mortem magnetic resonance imaging and quantitative histological study of multiple sclerosis pathology," Brain, vol. 135, no. 10, pp. 2938-2951, 2012.

[13] V. H. Perry and C. Holmes, "Microglial priming in neurodegenerative disease," Nature Reviews Neurology, vol. 10, no. 4, pp. 217-224, 2014.

[14] A. Kroner, N. Schwab, C. W. Ip et al., "Accelerated course of experimental autoimmune encephalomyelitis in PD-1-deficient central nervous system myelin mutants," The American Journal of Pathology, vol. 174, no. 6, pp. 2290-2299, 2009.

[15] J. J. G. Geurts, E.-J. Kooi, M. E. Witte, and P. van der Valk, "Multiple sclerosis as an 'inside-out' disease," Annals of Neurology, vol. 68, no. 5, pp. 767-768, 2010.

[16] P. K. Stys, G. W. Zamponi, J. van Minnen, and J. J. G. Geurts, "Will the real multiple sclerosis please stand up?" Nature Reviews Neuroscience, vol. 13, no. 7, pp. 507-514, 2012.

[17] M. H. Barnett and J. W. Prineas, "Relapsing and remitting multiple sclerosis: pathology of the newly forming lesion," Annals of Neurology, vol. 55, no. 4, pp. 458-468, 2004.

[18] K. J. Smith and H. Lassmann, "The role of nitric oxide in multiple sclerosis," The Lancet Neurology, vol. 1, no. 4, pp. 232241, 2002.

[19] M. W. Koch, G. S. M. Ramsaransing, A. V. Arutjunyan et al., "Oxidative stress in serum and peripheral blood leukocytes in patients with different disease courses of multiple sclerosis," Journal of Neurology, vol. 253, no. 4, pp. 483-487, 2006.

[20] O. Vladimirova, J. O’Connor, A. Cahill, H. Alder, C. Butunoi, and B. Kalman, "Oxidative damage to DNA in plaques of MS brains," Multiple Sclerosis, vol. 4, no. 5, pp. 413-418, 1998.

[21] K. P. Hammann and H. C. Hopf, "Monocytes constitute the only peripheral blood cell population showing an increased burst activity in multiple sclerosis patients," International Archives of Allergy and Applied Immunology, vol. 81, no. 3, pp. 230-234, 1986.
[22] A. Greco, L. Minghetti, G. Sette, C. Fieschi, and G. Levi, "Cerebrospinal fluid isoprostane shows oxidative stress in patients with multiple sclerosis," Neurology, vol. 53, no. 8, pp. 1876-1879, 1999.

[23] I. Nikić, D. Merkler, C. Sorbara et al., "A reversible form of axon damage in experimental autoimmune encephalomyelitis and multiple sclerosis," Nature Medicine, vol. 17, no. 4, pp. 495499, 2011.

[24] J. van Horssen, M. E. Witte, G. Schreibelt, and H. E. de Vries, "Radical changes in multiple sclerosis pathogenesis," Biochimica et Biophysica Acta, vol. 1812, no. 2, pp. 141-150, 2011.

[25] J. van Horssen, G. Schreibelt, J. Drexhage et al., "Severe oxidative damage in multiple sclerosis lesions coincides with enhanced antioxidant enzyme expression," Free Radical Biology \& Medicine, vol. 45, no. 12, pp. 1729-1737, 2008.

[26] L. Haider, M. T. Fischer, J. M. Frischer et al., "Oxidative damage in multiple sclerosis lesions," Brain, vol. 134, no. 7, pp. 1914-1924, 2011.

[27] M. Filippi, M. A. Rocca, F. Barkhof et al., "Association between pathological and MRI findings in multiple sclerosis," The Lancet Neurology, vol. 11, no. 4, pp. 349-360, 2012.

[28] D. H. Miller, P. Rudge, G. Johnson et al., "Serial gadolinium enhanced magnetic resonance imaging in multiple sclerosis," Brain, vol. 111, no. 4, pp. 927-939, 1988.

[29] M. I. Gaitán, C. D. Shea, I. E. Evangelou et al., "Evolution of the blood-brain barrier in newly forming multiple sclerosis lesions," Annals of Neurology, vol. 70, no. 1, pp. 22-29, 2011.

[30] H. Lassmann, "Review: the architecture of inflammatory demyelinating lesions: implications for studies on pathogenesis," Neuropathology and Applied Neurobiology, vol. 37, no. 7, pp. 698-710, 2011.

[31] J. W. Prineas and R. G. Wright, "Macrophages, lymphocytes, and plasma cells in the perivascular compartment in chronic multiple sclerosis," Laboratory Investigation, vol. 38, no. 4, pp. 409-421, 1978.

[32] A. H. Cross, P. T. Manning, R. M. Keeling, R. E. Schmidt, and T. P. Misko, "Peroxynitrite formation within the central nervous system in active multiple sclerosis," Journal of Neuroimmunology, vol. 88, no. 1-2, pp. 45-56, 1998.

[33] E. Gray, T. L. Thomas, S. Betmouni, N. Scolding, and S. Love, "Elevated myeloperoxidase activity in white matter in multiple sclerosis," Neuroscience Letters, vol. 444, no. 2, pp. 195-198, 2008.

[34] T. Zeis, A. Probst, A. J. Steck, C. Stadelmann, W. Brück, and N. Schaeren-Wiemers, "Molecular changes in white matter adjacent to an active demyelinating lesion in early multiple sclerosis: molecular changes in MS periplaque white matter," Brain Pathology, vol. 19, no. 3, pp. 459-466, 2009.

[35] M. T. Fischer, R. Sharma, J. L. Lim et al., "NADPH oxidase expression in active multiple sclerosis lesions in relation to oxidative tissue damage and mitochondrial injury," Brain, vol. 135, no. 3, pp. 886-899, 2012.

[36] K. J. Smith, "Newly lesioned tissue in multiple sclerosis-a role for oxidative damage?” Brain, vol. 134, no. 7, pp. 1877-1881, 2011.

[37] C. Schuh, I. Wimmer, S. Hametner et al., "Oxidative tissue injury in multiple sclerosis is only partly reflected in experimental disease models," Acta Neuropathologica, vol. 128, no. 2, pp. 247-266, 2014.

[38] B. M. Babior, J. D. Lambeth, and W. Nauseef, "The neutrophil NADPH oxidase," Archives of Biochemistry and Biophysics, vol. 397, no. 2, pp. 342-344, 2002. 
[39] S. Li, A. C. Vana, R. Ribeiro, and Y. Zhang, "Distinct role of nitric oxide and peroxynitrite in mediating oligodendrocyte toxicity in culture and in experimental autoimmune encephalomyelitis," Neuroscience, vol. 184, pp. 107-119, 2011.

[40] R. H. Hoek, S. R. Ruuls, C. A. Murphy et al., "Down-regulation of the macrophage lineage through interaction with OX2 (CD200)," Science, vol. 290, no. 5497, pp. 1768-1771, 2000.

[41] M. Gitik, S. Liraz-Zaltsman, P.-A. Oldenborg, F. Reichert, and S. Rotshenker, "Myelin down-regulates myelin phagocytosis by microglia and macrophages through interactions between CD47 on myelin and SIRP $\alpha$ (signal regulatory protein- $\alpha$ ) on phagocytes," Journal of Neuroinflammation, vol. 8, article 24, 2011.

[42] Y. Wang and H. Neumann, "Alleviation of neurotoxicity by microglial human Siglec-11," The Journal of Neuroscience, vol. 30, no. 9, pp. 3482-3488, 2010.

[43] R. M. Ransohoff and V. H. Perry, "Microglial physiology: unique stimuli, specialized responses," Annual Review of Immunology, vol. 27, pp. 119-145, 2009.

[44] V. H. Perry, J. A. R. Nicoll, and C. Holmes, "Microglia in neurodegenerative disease," Nature Reviews Neurology, vol. 6, no. 4, pp. 193-201, 2010.

[45] H. Konno, T. Yamamoto, H. Suzuki et al., "Targeting of adoptively transferred experimental allergic encephalitis lesion at the sites of Wallerian degeneration," Acta Neuropathologica, vol. 80, no. 5, pp. 521-526, 1990.

[46] L. Haider, C. Simeonidou, G. Steinberger et al., "Multiple sclerosis deep grey matter: the relation between demyelination, neurodegeneration, inflammation and iron," Journal of Neurology, Neurosurgery and Psychiatry, vol. 85, pp. 1386-1395, 2014.

[47] B. Halliwell, "Role of free radicals in the neurodegenerative diseases: therapeutic implications for antioxidant treatment," Drugs \& Aging, vol. 18, no. 9, pp. 685-716, 2001.

[48] S. W. Hulet, E. J. Hess, W. Debinski et al., "Characterization and distribution of ferritin binding sites in the adult mouse brain," Journal of Neurochemistry, vol. 72, no. 2, pp. 868-874, 1999.

[49] S. W. Hulet, S. Powers, and J. R. Connor, "Distribution of transferrin and ferritin binding in normal and multiple sclerotic human brains," Journal of the Neurological Sciences, vol. 165, no. 1, pp. 48-55, 1999.

[50] B. Hallgren and P. Sourander, "The effect of age on the nonhaemin iron in the human brain," Journal of Neurochemistry, vol. 3, no. 1, pp. 41-51, 1958.

[51] K. I. Rathore, B. J. Kerr, A. Redensek et al., "Ceruloplasmin protects injured spinal cord from iron-mediated oxidative damage," The Journal of Neuroscience, vol. 28, no. 48, pp. 1273612747, 2008.

[52] S. Hametner, I. Wimmer, L. Haider, S. Pfeifenbring, W. Brück, and H. Lassmann, "Iron and neurodegeneration in the multiple sclerosis brain," Annals of Neurology, vol. 74, no. 6, pp. 848-861, 2013.

[53] K. O. Lopes, D. L. Sparks, and W. J. Streit, "Microglial dystrophy in the aged and Alzheimer's disease brain is associated with ferritin immunoreactivity," Glia, vol. 56, no. 10, pp. 1048-1060, 2008.

[54] W. Craelius, M. W. Migdal, C. P. Luessenhop, A. Sugar, and I. Mihalakis, "Iron deposits surrounding multiple sclerosis plaques," Archives of Pathology \& Laboratory Medicine, vol. 106, no. 8, pp. 397-399, 1982.

[55] F. Bagnato, S. Hametner, B. Yao et al., “Tracking iron in multiple sclerosis: a combined imaging and histopathological study at 7 Tesla," Brain, vol. 134, no. 12, pp. 3599-3612, 2011.
[56] M. A. Friese, B. Schattling, and L. Fugger, "Mechanisms of neurodegeneration and axonal dysfunction in multiple sclerosis," Nature Reviews Neurology, vol. 10, no. 4, pp. 225-238, 2014.

[57] H. Lassmann, J. Van Horssen, and D. Mahad, "Progressive multiple sclerosis: Pathology and pathogenesis," Nature Reviews Neurology, vol. 8, no. 11, pp. 647-656, 2012.

[58] H. Spatz, "Über den eisennachweis im gehirn, besonders in zentren des extrapyramidal-motorischen systems. I. Teil," Zeitschrift für die Gesamte Neurologie und Psychiatrie, vol. 77, no. 1, pp. 261-390, 1922.

[59] M. Vercellino, S. Masera, M. Lorenzatti et al., "Demyelination, inflammation, and neurodegeneration in multiple sclerosis deep gray matter," Journal of Neuropathology and Experimental Neurology, vol. 68, no. 5, pp. 489-502, 2009.

[60] A. Cifelli, M. Arridge, P. Jezzard, M. M. Esiri, J. Palace, and P. M. Matthews, "Thalamic neurodegeneration in multiple sclerosis," Annals of Neurology, vol. 52, no. 5, pp. 650-653, 2002.

[61] S. Murphy, "Production of nitric oxide by glial cells: Regulation and potential roles in the CNS," Glia, vol. 29, no. 1, pp. 1-13, 2000.

[62] M. T. Heneka and D. L. Feinstein, "Expression and function of inducible nitric oxide synthase in neurons," Journal of Neuroimmunology, vol. 114, no. 1-2, pp. 8-18, 2001.

[63] C. E. Cooper and G. C. Brown, "The interactions between nitric oxide and brain nerve terminals as studied by electron paramagnetic resonance," Biochemical and Biophysical Research Communications, vol. 212, no. 2, pp. 404-412, 1995.

[64] P. Mander, V. Borutaite, S. Moncada, and G. C. Brown, "Nitric oxide from inflammatory-activated glia synergizes with hypoxia to induce neuronal death," Journal of Neuroscience Research, vol. 79, no. 1-2, pp. 208-215, 2005.

[65] A. Bal-Price and G. C. Brown, "Inflammatory neurodegeneration mediated by nitric oxide from activated glia-inhibiting neuronal respiration, causing glutamate release and excitotoxicity," The Journal of Neuroscience, vol. 21, no. 17, pp. 6480-6491, 2001.

[66] G. C. Brown, "Mechanisms of inflammatory neurodegeneration: INOS and NADPH oxidase," Biochemical Society Transactions, vol. 35, no. 5, pp. 1119-1121, 2007.

[67] S. Cho, E.-M. Park, P. Zhou, K. Frys, M. E. Ross, and C. Iadecola, "Obligatory role of inducible nitric oxide synthase in ischemic preconditioning," Journal of Cerebral Blood Flow and Metabolism, vol. 25, no. 4, pp. 493-501, 2005.

[68] V. Mehta, W. Pei, G. Yang et al., "Iron is a sensitive biomarker for inflammation in multiple sclerosis lesions," PLoS ONE, vol. 8, no. 3, Article ID e57573, 2013.

[69] K. H. Lee, S. J. Yun, K. N. Nam, Y. S. Gho, and E. H. Lee, "Activation of microglial cells by ceruloplasmin," Brain Research, vol. 1171, no. 1, pp. 1-8, 2007.

[70] I. Kawachi, "Deep grey matter involvement in multiple sclerosis: key player or bystander?" Journal of Neurology, Neurosurgery and Psychiatry, vol. 85, no. 12, p. 1293, 2014.

[71] W. E. Klunk, J. C. Price, C. A. Mathis et al., "Amyloid deposition begins in the striatum of presenilin-1 mutation carriers from two unrelated pedigrees," The Journal of Neuroscience, vol. 27, no. 23, pp. 6174-6184, 2007.

[72] N. S. Ryan, S. Keihaninejad, T. J. Shakespeare et al., "Magnetic resonance imaging evidence for presymptomatic change in thalamus and caudate in familial Alzheimer's disease," Brain, vol. 136, part 5, pp. 1399-1414, 2013.

[73] S. Love, D. Louis, and D. W. Ellison, Greenfield's Neuropathology, CRC Press, 2008. 
[74] S. DiMauro, E. A. Schon, V. Carelli, and M. Hirano, "The clinical maze of mitochondrial neurology," Nature Reviews Neurology, vol. 9, no. 8, pp. 429-444, 2013.

[75] N. K. Olsen, A. W. Hansen, S. Norby, A. L. Edal, J. R. Jorgensen, and T. Rosenberg, "Leber's hereditary optic neuropathy associated with a disorder indistinguishable from multiple sclerosis in a male harbouring the mitochondrial DNA 11778 mutation," Acta Neurologica Scandinavica, vol. 91, no. 5, pp. 326-329, 1995.

[76] M. E. Witte, D. J. Mahad, H. Lassmann, and J. van Horssen, "Mitochondrial dysfunction contributes to neurodegeneration in multiple sclerosis," Trends in Molecular Medicine, vol. 20, no. 3, pp. 179-187, 2014.

[77] C. Lucchinetti, W. Brück, J. Parisi, B. Scheithauer, M. Rodriguez, and H. Lassmann, "Heterogeneity of multiple sclerosis lesions: Implications for the pathogenesis of demyelination," Annals of Neurology, vol. 47, no. 6, pp. 707-717, 2000.

[78] D. Mahad, I. Ziabreva, H. Lassmann, and D. Turnbull, "Mitochondrial defects in acute multiple sclerosis lesions," Brain, vol. 131, no. 7, pp. 1722-1735, 2008.

[79] R. Dutta, J. McDonough, X. Yin et al., "Mitochondrial dysfunction as a cause of axonal degeneration in multiple sclerosis patients," Annals of Neurology, vol. 59, no. 3, pp. 478-489, 2006.

[80] G. R. Campbell, I. Ziabreva, A. K. Reeve et al., "Mitochondrial DNA deletions and neurodegeneration in multiple sclerosis," Annals of Neurology, vol. 69, no. 3, pp. 481-492, 2011.

[81] B. M. Babior, "NADPH oxidase," Current Opinion in Immunology, vol. 16, no. 1, pp. 42-47, 2004.

[82] A. Jekabsone, J. J. Neher, V. Borutaite, and G. C. Brown, "Nitric oxide from neuronal nitric oxide synthase sensitises neurons to hypoxia-induced death via competitive inhibition of cytochrome oxidase," Journal of Neurochemistry, vol. 103, no. 1, pp. 346-356, 2007.

[83] F. M. Yakes and B. Van Houten, "Mitochondrial DNA damage is more extensive and persists longer than nuclear DNA damage in human cells following oxidative stress," Proceedings of the National Academy of Sciences of the United States of America, vol. 94, no. 2, pp. 514-519, 1997.

[84] P. Mao and P. H. Reddy, "Is multiple sclerosis a mitochondrial disease?" Biochimica et Biophysica Acta, vol. 1802, no. 1, pp. 6679, 2010.

[85] M. E. Witte, J. J. G. Geurts, H. E. de Vries, P. van der Valk, and J. van Horssen, "Mitochondrial dysfunction: a potential link between neuroinflammation and neurodegeneration?" Mitochondrion, vol. 10, no. 5, pp. 411-418, 2010.

[86] B. Halliwell and J. M. C. Gutteridge, "Oxygen toxicity, oxygen radicals, transition metals and disease," The Biochemical Journal, vol. 219, no. 1, pp. 1-14, 1984.

[87] E. Cadenas, A. Boveris, C. I. Ragan, and A. O. M. Stoppani, "Production of superoxide radicals and hydrogen peroxide by NADH ubiquinone reductase and ubiquinol cytochrome $\mathrm{c}$ reductase from beef heart mitochondria," Archives of Biochemistry and Biophysics, vol. 180, no. 2, pp. 248-257, 1977.

[88] C. Nathan and A. Ding, "Snapshot: Reactive oxygen intermediates (ROI)," Cell, vol. 140, no. 6, pp. 951-951.e2, 2010.

[89] T. Klimova and N. S. Chandel, "Mitochondrial complex III regulates hypoxic activation of HIF," Cell Death and Differentiation, vol. 15, no. 4, pp. 660-666, 2008.

[90] M. P. Murphy, "How mitochondria produce reactive oxygen species," The Biochemical Journal, vol. 417, no. 1, pp. 1-13, 2009.

[91] D. C. Wallace, "Mitochondrial DNA mutations in disease and aging," Environmental and Molecular Mutagenesis, vol. 51, no. 5, pp. $440-450,2010$.
[92] X. Qi, A. S. Lewin, L. Sun, W. W. Hauswirth, and J. Guy, "Mitochondrial protein nitration primes neurodegeneration in experimental autoimmune encephalomyelitis," The Journal of Biological Chemistry, vol. 281, no. 42, pp. 31950-31962, 2006.

[93] N.-G. Larsson, "Somatic mitochondrial DNA mutations in mammalian aging," Annual Review of Biochemistry, vol. 79, pp. 683-706, 2010.

[94] H. Harris and D. C. Rubinsztein, "Control of autophagy as a therapy for neurodegenerative disease," Nature Reviews Neurology, vol. 8, no. 2, pp. 108-117, 2012.

[95] B. Ravikumar, S. Sarkar, J. E. Davies et al., "Regulation of mammalian autophagy in physiology and pathophysiology," Physiological Reviews, vol. 90, no. 4, pp. 1383-1435, 2010.

[96] P. K. Stys, "General mechanisms of axonal damage and its prevention," Journal of the Neurological Sciences, vol. 233, no. 1-2, pp. 3-13, 2005.

[97] B. D. Trapp and P. K. Stys, "Virtual hypoxia and chronic necrosis of demyelinated axons in multiple sclerosis," The Lancet Neurology, vol. 8, no. 3, pp. 280-291, 2009.

[98] B. Kornek, M. K. Storch, R. Weissert et al., "Multiple sclerosis and chronic autoimmune encephalomyelitis: a comparative quantitative study of axonal injury in active, inactive, and remyelinated lesions," American Journal of Pathology, vol. 157, no. 1, pp. 267-276, 2000.

[99] P. Werner, D. Pitt, and C. S. Raine, "Multiple sclerosis: altered glutamate homeostasis in lesions correlates with oligodendrocyte and axonal damage," Annals of Neurology, vol. 50, no. 2, pp. 169-180, 2001.

[100] S. Vergo, M. J. Craner, R. Etzensperger et al., "Acid-sensing ion channel 1 is involved in both axonal injury and demyelination in multiple sclerosis and its animal model," Brain, vol. 134, part 2, pp. 571-584, 2011.

[101] S. G. Waxman, "Mechanisms of disease: sodium channels and neuroprotection in multiple sclerosis-current status," Nature Clinical Practice Neurology, vol. 4, no. 3, pp. 159-169, 2008.

[102] J. Herschel, "A scaled ratio of body weight to brain weight of a comparative index for relative importance of brain size in mammals of widely varying body mass," Psychological Reports, vol. 31, no. 1, pp. 84-86, 1972.

[103] U. Graumann, R. Reynolds, A. J. Steck, and N. SchaerenWiemers, "Molecular changes in normal appearing white matter in multiple sclerosis are characteristic of neuroprotective mechanisms against hypoxic insult," Brain Pathology, vol. 13, no. 4, pp. 554-573, 2003.

[104] F. Aboul-Enein, H. Rauschka, B. Kornek et al., "Preferential loss of myelin-associated glycoprotein reflects hypoxia-like white matter damage in stroke and inflammatory brain diseases," Journal of Neuropathology and Experimental Neurology, vol. 62, no. 1, pp. 25-33, 2003.

[105] T. Zeis, U. Graumann, R. Reynolds, and N. Schaeren-Wiemers, "Normal-appearing white matter in multiple sclerosis is in a subtle balance between inflammation and neuroprotection," Brain, vol. 131, no. 1, pp. 288-303, 2008.

[106] C. Stadelmann, S. Ludwin, T. Tabira et al., "Tissue preconditioning may explain concentric lesions in Baló's type of multiple sclerosis," Brain, vol. 128, no. 5, pp. 979-987, 2005.

[107] A. N. Mháille, S. McQuaid, A. Windebank et al., "Increased expression of endoplasmic reticulum stress-related signaling pathway molecules in multiple sclerosis lesions," Journal of Neuropathology and Experimental Neurology, vol. 67, no. 3, pp. 200-211, 2008. 
[108] J. M. McMahon, S. McQuaid, R. Reynolds, and U. F. Fitzgerald, "Increased expression of ER stress- and hypoxia-associated molecules in grey matter lesions in multiple sclerosis," Multiple Sclerosis, vol. 18, no. 10, pp. 1437-1447, 2012.

[109] W. T. Regenold, P. Phatak, M. J. Makley, R. D. Stone, and M. A. Kling, "Cerebrospinal fluid evidence of increased extramitochondrial glucose metabolism implicates mitochondrial dysfunction in multiple sclerosis disease progression," Journal of the Neurological Sciences, vol. 275, no. 1-2, pp. 106-112, 2008.

[110] C. A. Davie, C. P. Hawkins, G. J. Barker et al., "Serial proton magnetic resonance spectroscopy in acute multiple sclerosis lesions," Brain, vol. 117, no. 1, pp. 49-58, 1994.

[111] T. Haufschild, S. G. Shaw, J. Kesselring, and J. Flammer, "Increased endothelin-1 plasma levels in patients with multiple sclerosis," Journal of Neuro-Ophthalmology, vol. 21, no. 1, pp. 3738, 2001.

[112] M. Pache, H. J. Kaiser, N. Akhalbedashvili et al., "Extraocular blood flow and endothelin-1 plasma levels in patients with multiple sclerosis," European Neurology, vol. 49, no. 3, pp. 164168, 2003.

[113] A. L. Davies, R. A. Desai, P. S. Bloomfield et al., "Neurological deficits caused by tissue hypoxia in neuroinflammatory disease," Annals of Neurology, vol. 74, no. 6, pp. 815-825, 2013.

[114] B. Brownell and J. T. Hughes, "The distribution of plaques in the cerebrum in multiple sclerosis," Journal of Neurology, Neurosurgery, and Psychiatry, vol. 25, pp. 315-320, 1962.

[115] C. M. Holland, A. Charil, I. Csapo et al., "The relationship between normal cerebral perfusion patterns and white matter lesion distribution in 1,249 patients with multiple sclerosis," Journal of Neuroimaging, vol. 22, no. 2, pp. 129-136, 2012.

[116] M. A. Lee, S. Smith, J. Palace et al., "Spatial mapping of T2 and gadolinium-enhancing T1 lesion volumes in multiple sclerosis: evidence for distinct mechanisms of lesion genesis?" Brain, vol. 122, part 7, pp. 1261-1270, 1999.

[117] J. Lansley, D. Mataix-Cols, M. Grau, J. Radua, and J. SastreGarriga, "Localized grey matter atrophy in multiple sclerosis: a meta-analysis of voxel-based morphometry studies and associations with functional disability," Neuroscience and Biobehavioral Reviews, vol. 37, no. 5, pp. 819-830, 2013.

[118] N. Jafari and R. Q. Hintzen, “The association between cigarette smoking and multiple sclerosis," Journal of the Neurological Sciences, vol. 311, no. 1-2, pp. 78-85, 2011.

[119] M. A. Hernán, M. J. Olek, and A. Ascherio, "Cigarette smoking and incidence of multiple sclerosis," American Journal of Epidemiology, vol. 154, no. 1, pp. 69-74, 2001.

[120] M. Emre and C. de Decker, "Effects of cigarette smoking on motor functions in patients with multiple sclerosis," Archives of Neurology, vol. 49, no. 12, pp. 1243-1247, 1992.

[121] D. F. Church and W. A. Pryor, "Free-radical chemistry of cigarette smoke and its toxicological implications," Environmental Health Perspectives, vol. 64, pp. 111-126, 1985.

[122] W. C. Butts, M. Kuehneman, and G. M. Widdowson, "Automated method for determining serum thiocyanate, to distinguish smokers from nonsmokers," Clinical Chemistry, vol. 20, no. 10, pp. 1344-1348, 1974.

[123] J. Zambonin, S. Engeham, G. R. Campbell et al., "Identification and investigation of mitochondria lacking cytochrome c oxidase activity in axons," Journal of Neuroscience Methods, vol. 192, no. 1, pp. 115-120, 2010.
[124] I. Tellez, D. Johnson, R. L. Nagel, and A. Cerami, "Neurotoxicity of sodium cyanate. New pathological and ultrastructural observations in Macaca nemestrina," Acta Neuropathologica, vol. 47, no. 1, pp. 75-79, 1979.

[125] D. J. Philbrick, J. B. Hopkins, D. C. Hill, J. C. Alexander, and R. G. Thomson, "Effects of prolonged cyanide and thiocyanate feeding in rats," Journal of Toxicology and Environmental Health, vol. 5, no. 4, pp. 579-592, 1979.

[126] A. Ohnishi, C. M. Peterson, and P. J. Dyck, "Axonal degeneration in sodium cyanate induced neuropathy," Archives of Neurology, vol. 32, no. 8, pp. 530-534, 1975.

[127] H. D. Jonez, "Multiple sclerosis; treatment with histamine and d-tubocurarine," Annals of allergy, vol. 6, no. 5, pp. 550-563, 1948.

[128] R. M. Brickner, "Pharmacological reduction of abnormality in multiple sclerosis within minutes: a statistical study," The Journal of Nervous and Mental Disease, vol. 127, no. 4, pp. 308322, 1958.

[129] B. T. Horton, “The clinical use of histamine," Postgraduate Medicine, vol. 9, no. 1, pp. 1-10, 1951.

[130] B. H. Fischer, M. Marks, and T. Reich, "Hyperbaric-oxygen treatment of multiple sclerosis. A randomized, placebocontrolled, double-blind study," The New England Journal of Medicine, vol. 308, no. 4, pp. 181-186, 1983.

[131] M. Bennett and R. Heard, "Hyperbaric oxygen therapy for multiple sclerosis," CNS Neuroscience \& Therapeutics, vol. 16, no. 2, pp. 115-124, 2010.

[132] J. M. McCord, "Oxygen-derived free radicals in postischemic tissue injury," The New England Journal of Medicine, vol. 312, no. 3, pp. 159-163, 1985.

[133] L. di Nuzzo, R. Orlando, C. Nasca, and F. Nicoletti, "Molecular pharmacodynamics of new oral drugs used in the treatment of multiple sclerosis," Drug Design, Development and Therapy, vol. 8, pp. 555-568, 2014.

[134] E. Hondares, O. Mora, P. Yubero et al., "Thiazolidinediones and rexinoids induce peroxisome proliferator-activated receptorcoactivator (PGC)-1alpha gene transcription: an autoregulatory loop controls PGC-lalpha expression in adipocytes via peroxisome proliferator-activated receptor-gamma coactivation," Endocrinology, vol. 147, no. 6, pp. 2829-2838, 2006.

[135] K. S. Shindler, E. Ventura, M. Dutt, P. Elliott, D. C. Fitzgerald, and A. Rostami, "Oral resveratrol reduces neuronal damage in a model of multiple sclerosis," Journal of Neuro-Ophthalmology, vol. 30, no. 4, pp. 328-339, 2010.

[136] M. E. Witte, P. G. Nijland, J. A. R. Drexhage et al., "Reduced expression of PGC- $1 \alpha$ partly underlies mitochondrial changes and correlates with neuronal loss in multiple sclerosis cortex," Acta Neuropathologica, vol. 125, no. 2, pp. 231-243, 2013.

[137] M. Forte, B. G. Gold, G. Marracci et al., "Cyclophilin $\mathrm{D}$ inactivation protects axons in experimental autoimmune encephalomyelitis, an animal model of multiple sclerosis," Proceedings of the National Academy of Sciences of the United States of America, vol. 104, no. 18, pp. 7558-7563, 2007.

[138] K. Su, D. Bourdette, and M. Forte, "Genetic inactivation of mitochondria-targeted redox enzyme p66shcA preserves neuronal viability and mitochondrial integrity in response to oxidative challenges," Frontiers in Physiology, vol. 3, article 285, 2012.

[139] X. Qi, A. S. Lewin, L. Sun, W. W. Hauswirth, and J. Guy, "Suppression of mitochondrial oxidative stress provides long-term neuroprotection in experimental optic neuritis," Investigative 
Ophthalmology \& Visual Science, vol. 48, no. 2, pp. 681-691, 2007.

[140] P. Mao, M. Manczak, U. P. Shirendeb, and P. H. Reddy, "MitoQ, a mitochondria-targeted antioxidant, delays disease progression and alleviates pathogenesis in an experimental autoimmune encephalomyelitis mouse model of multiple sclerosis," Biochimica et Biophysica Acta-Molecular Basis of Disease, vol. 1832, no. 12, pp. 2322-2331, 2013.

[141] S. Sawcer, G. Hellenthal, M. Pirinen et al., "Genetic risk and a primary role for cell-mediated immune mechanisms in multiple sclerosis," Nature, vol. 476, no. 7359, pp. 214-219, 2011.

[142] M. Prinz, H. Schmidt, A. Mildner et al., "Distinct and nonredundant in vivo functions of IFNAR on myeloid cells limit autoimmunity in the central nervous system," Immunity, vol. 28, no. 5, pp. 675-686, 2008.

[143] M. Comabella, J. D. Lünemann, J. Río et al., "A type I interferon signature in monocytes is associated with poor response to interferon- $\beta$ in multiple sclerosis," Brain, vol. 132, no. 12, pp. 3353-3365, 2009.

[144] P. H. Lalive, O. Neuhaus, M. Benkhoucha et al., "Glatiramer acetate in the treatment of multiple sclerosis: emerging concepts regarding its mechanism of action," CNS Drugs, vol. 25, no. 5, pp. 401-414, 2011.

[145] H.-P. Hartung, R. Gonsette, N. König et al., "Mitoxantrone in progressive multiple sclerosis: a placebo-controlled, doubleblind, randomised, multicentre trial," The Lancet, vol. 360, no. 9350, pp. 2018-2025, 2002.

[146] R. A. Linker, D.-H. Lee, S. Ryan et al., "Fumaric acid esters exert neuroprotective effects in neuroinflammation via activation of the Nrf2 antioxidant pathway," Brain, vol. 134, no. 3, pp. 678692, 2011.

[147] V. E. Miron, S. K. Ludwin, P. J. Darlington et al., "Fingolimod (FTY720) enhances remyelination following demyelination of organotypic cerebellar slices," The American Journal of Pathology, vol. 176, no. 6, pp. 2682-2694, 2010.

[148] L. Kappos, H. Hartung, M. S. Freedman et al., "Atacicept in multiple sclerosis (ATAMS): a randomised, placebo-controlled, double-blind, phase 2 trial," The Lancet Neurology, vol. 13, no. 4, pp. 353-363, 2014.

[149] A. P. Gregory, C. A. Dendrou, K. E. Attfield et al., "TNF receptor 1 genetic risk mirrors outcome of anti-TNF therapy in multiple sclerosis," Nature, vol. 488, no. 7412, pp. 508-511, 2012.

[150] B. M. Segal, C. S. Constantinescu, A. Raychaudhuri, L. Kim, R. Fidelus-Gort, and L. H. Kasper, "Repeated subcutaneous injections of IL12/23 p40 neutralising antibody, ustekinumab, in patients with relapsing-remitting multiple sclerosis: a phase II, double-blind, placebo-controlled, randomised, dose-ranging study," The Lancet Neurology, vol. 7, no. 9, pp. 796-804, 2008.

[151] A. Haghikia, R. Hohlfeld, R. Gold, and L. Fugger, “Therapies for multiple sclerosis: translational achievements and outstanding needs," Trends in Molecular Medicine, vol. 19, no. 5, pp. 309-319, 2013.

[152] R. Gold, L. Kappos, D. L. Arnold et al., "Placebo-controlled phase 3 study of oral BG-12 for relapsing multiple sclerosis," The New England Journal of Medicine, vol. 367, no. 12, pp. 1098-1107, 2012. 


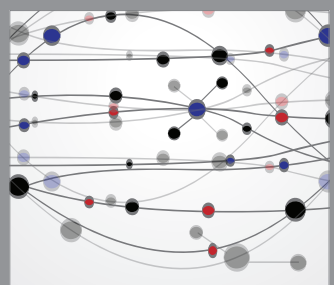

The Scientific World Journal
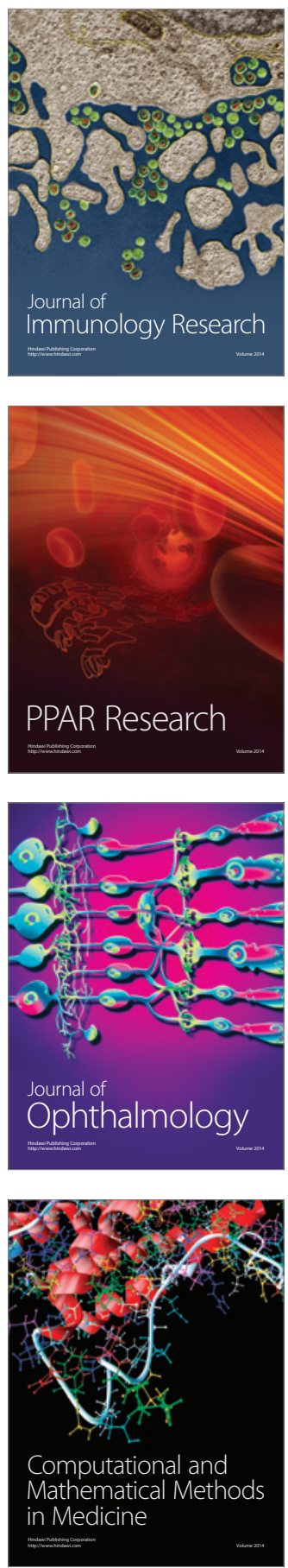

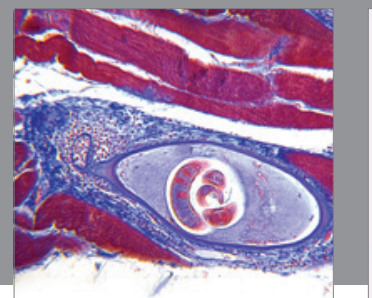

Gastroenterology

Research and Practice
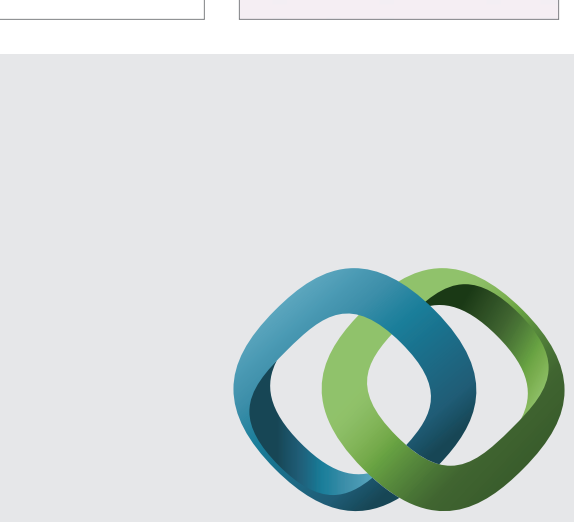

\section{Hindawi}

Submit your manuscripts at

http://www.hindawi.com
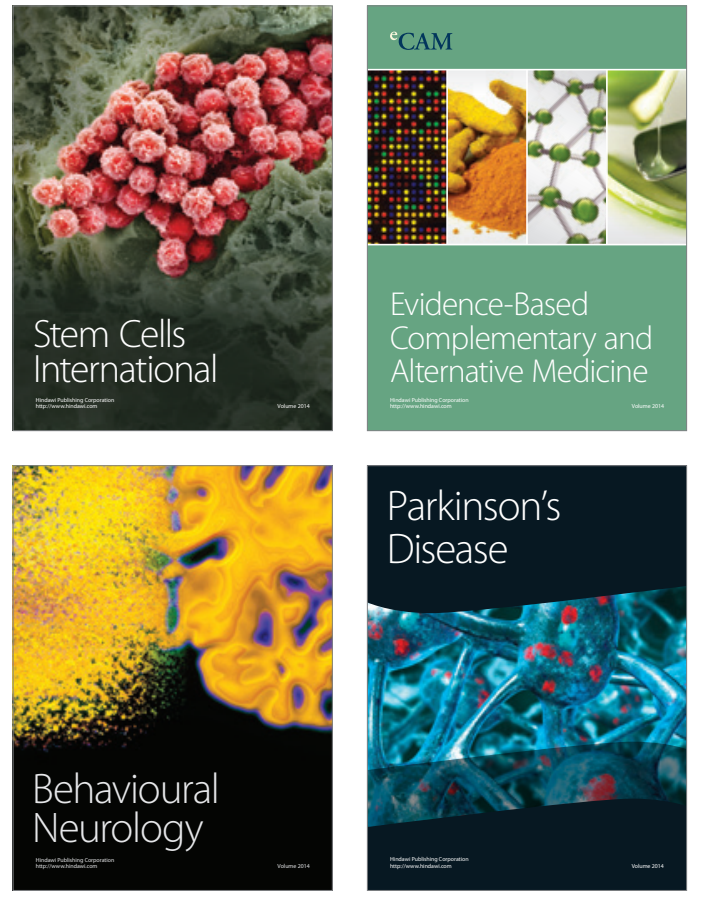
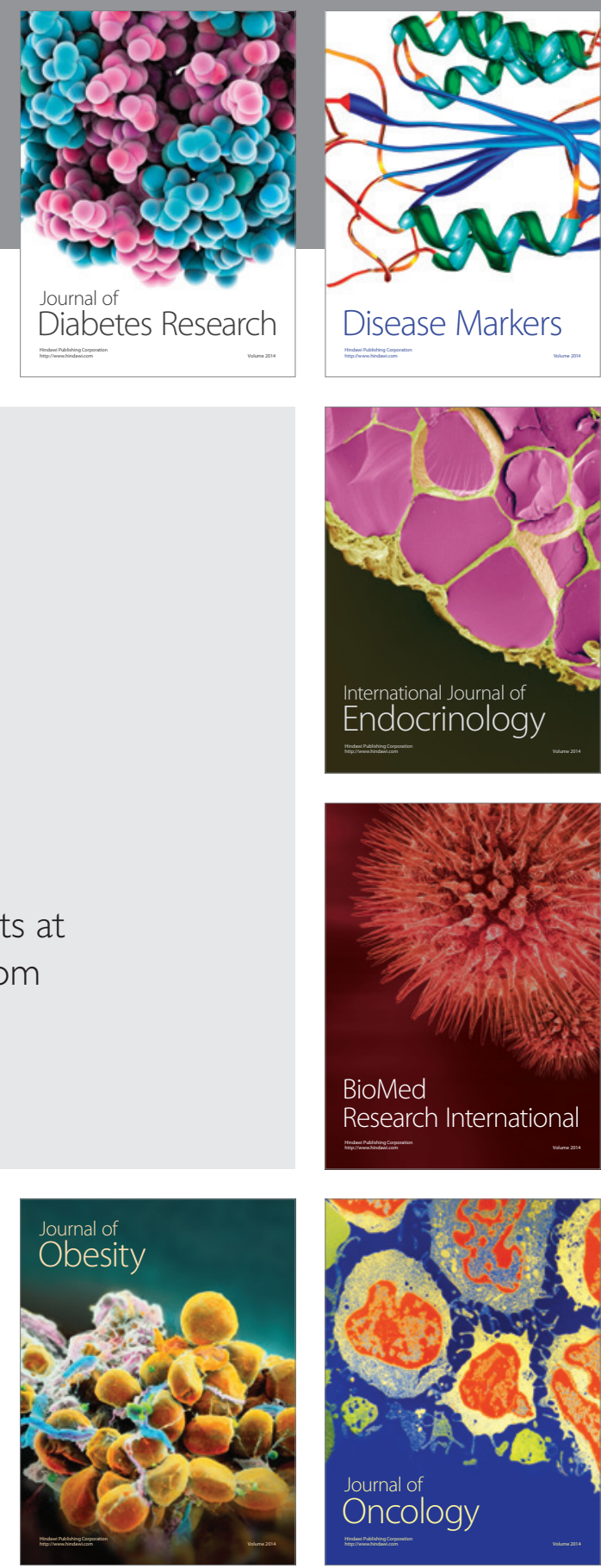

Disease Markers
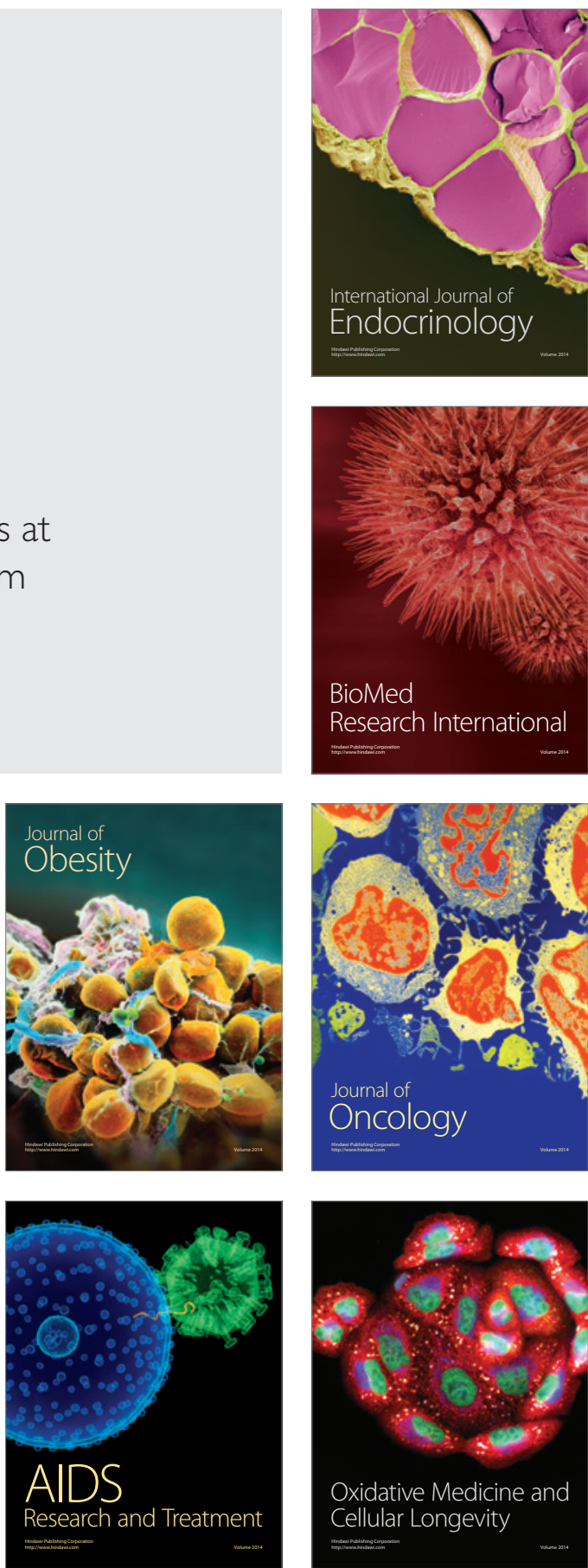